\title{
A study of crossover operator in real coded genetic algorithm
}

\author{
OMinoru ITO \\ Masanori SUGISAKA \\ Faculty of Engineering, Oita university \\ 700, Oaza Dannoharu, Oita, 870-1192, JAPAN \\ Tel : 81-97-554-7831 \\ Fax : 81-97-554-7841 \\ minoru@cc.oita-u.ac.jp \\ msugi@cc.oita-u.ac.jp
}

\begin{abstract}
In this paper, we propose a new crossover operator for real coded genetic algorithm (real-coded GA). Real-coded GA is expected to be powerful optimization technique for the nonlinear functions. A designing of crossover operator is important for a GA with good performance. At this point, for realcoded GA, many crossover operators are proposed. As a major crossover, the BLX- $\alpha$ proposed by Eshelman et al., the unimodal normal distribution crossover (UNDX) proposed by Ono et al.. However, the conventional crossover operators have merits and demerits of the accuracy and the processing speed. Then, in this paper, we propose the center distribution crossover (CDX) as a new crossover operator which improves the above problems. The performance of the proposed method is evaluated with the standard test functions. The performance of the proposed method are compared with the conventional methods.
\end{abstract}

Keywords: Genetic Algorithm, Real-Coded GA, Crossover operator, Function Optimization

\section{Introduction}

Genetic Algorithm (GA) is a stochastic search and an optimization algorithm. The GA is based on the principles of biological evolution $[1-3,5]$. The GA searches for a solution using the selection operator, crossover operator and mutation operator in multiple searching points. The GA is applied to many optimization problems and the effectiveness are reported [4].

Recently, in this GA, real-coded GA attract the attention as an optimization methods for the nonlinear functions and the effectiveness are reported [6]. For real-coded GA, there have been proposed many crossover operators so far. As a major crossover operator, the BLX- $\alpha$ proposed by Eshelman et al. [7], the unimodal normal crossover (UNDX) proposed by Ono et al. $[8,9]$.

However, the above crossover operators have merits and demerits of the accuracy and the processing speed. Then, in this paper, we propose the center distribution crossover (CDX) as a new crossover operator which improves the above problems. We also show the efficiency of the proposed method by applying to the standard test functions [13].

\section{Real-coded genetic algorithm}

In this section, we review the UNDX and the MGG model as a basic crossover operator and generation alternation model for real-coded GA.

\subsection{UNDX}

The UNDX proposed by Ono et al. $[8,9]$ shows good performance in theoptimization problems. The UNDX generates children obeying a normal distribution around the parents as shown in Fig1. The normal distribution is centered at the middle point of parents $x^{1}$ and $x^{2}$. The standard deviation is proportional to the distance between parents $x^{1}$ and $x^{2}$ in the direction of the line connecting the parents. Moreover, the standard deviation in the verticality directions are proportional to the distance of the parent $x^{3}$ and the line connecting the parents $x^{1}$ and $x^{2}$.

The mathematical descriptions of the UNDX show the following : where $x^{c}$ is child, and $x^{p}$ is the center of parents $x^{1}$ and $x^{2}$, and $D$ is distance from parent $x^{3}$ to the line connecting the parents 


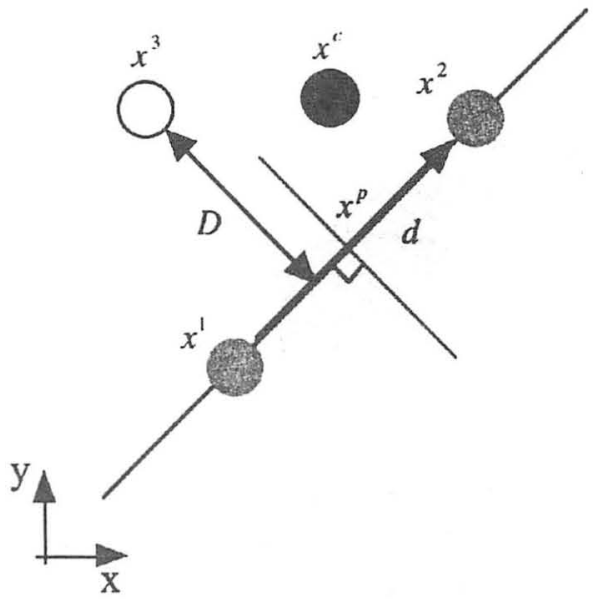

Fig 1: Unimodal Normal Distribution Crossover, UNDX

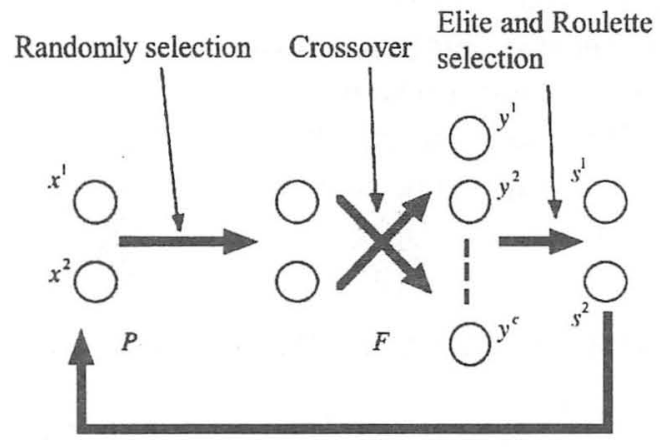

Fig 2: Minimal Generation Gap, MGG

$x^{1}$ and $x^{2}$, and $\xi$ and $\eta_{i}$ are normal distribution random number, and $\boldsymbol{e}_{i}$ is the unit vector.

$$
\begin{gathered}
x^{c}=x^{p}+\xi d+D \sum_{i=1}^{n-1} \eta_{i} e_{i} \\
x^{p}=\left(x^{1}+x^{2}\right) / 2 \\
d=x^{1}-x^{2} \\
\xi \sim N\left(0, \sigma_{\xi}^{2}\right), \eta_{i} \sim N\left(0, \sigma_{\eta}^{2}\right)
\end{gathered}
$$

\subsection{MGG}

The MGG model proposed by Satoh et al. [11,12] is an effective one of generation alternation models. In the MGG model, we randomly select parents from population, and we generate children by crossover. From the parents and their children, we select an elite individual and a random one using the roulette selection based on the fitness value. The original parents are replaced by the selected children. In this model, the original parents are two individuals, and replacing individuals are also two. Fig2 shows the outline of the MGG model.

\section{Proposed method}

\subsection{Center Distribution Crossover, CDX}

We show the outline of the CDX as the proposed method. In the CDX, $n+1$ parents are selected from the population and $m$ children are generated around the center of parents.

The mathematical descriptions of CDX show the following : where $C$ is child, and $G$ is the mean of parents, and D is average distance from $G$ to each parents, and $\eta_{i}$ is normal distribution random number (average is 0 , variance is $\sigma_{\eta}^{2}$ ), and $\boldsymbol{e}_{i}$ is the unit vector, and $\boldsymbol{x}_{\boldsymbol{i}}$ is parental vector.

$$
\begin{gathered}
C=G+D \sum_{i=1}^{n+1} \eta_{i} e_{i} \\
G=\frac{1}{n+1} \sum_{i=1}^{n+1} x_{i} \\
D=\frac{1}{n+1} \sum_{i=1}^{n+1}\left|G-x_{i}\right| \\
\eta_{i} \sim N\left(0, \sigma_{\eta}^{2}\right)
\end{gathered}
$$

In the conventional method, depending on how we select parents, it is easy to bias a searching area. On the other, the CDX effectively avoids a bias about a searching area. From the above point, the CDX effectively aoids a stagnation of a searhing solution.

\subsection{The configuration of GA with the CDX}

We show the configuration of the real-coded GA with the proposed method. We use the MGG model as a generation alternation model. However, the proposed method uses many parents than the UNDX. We adjust the MGG model as below. In addition, Fig3 shows the outline.

1. We randomly select $n+1$ parents from populations. 


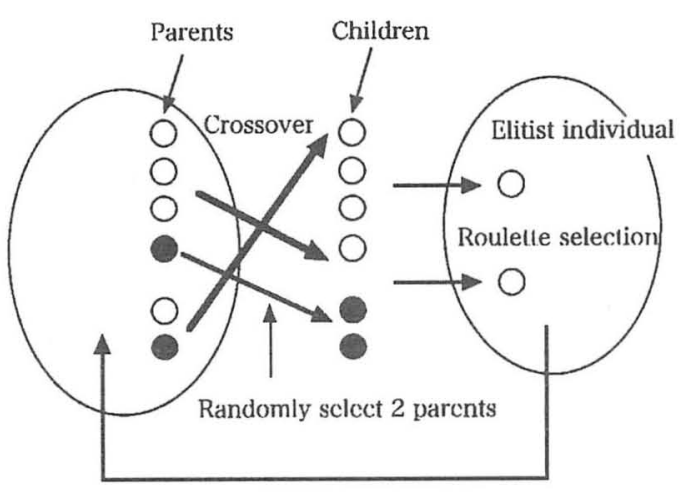

Fig 3: The configuration of GA with the CDX

2. We generate $m$ children by crossover.

3 . We randomly select 2 individuals from parents.

4. From the above 2 parents and $m$ children, we select an elite individual and a random one using the roulette selection, and the 2 parents are replaced by the selected individuals.

\section{Simulation and Results}

In order to confirm the effectiveness of the proposed method, we compare the proposed method with the UNDX. In the first subsection, we show the test functions. In the second subsection, we show the experimental setting. In the last subsection, we show the simulation results and discuss the simulation results.

\subsection{Test functions}

In this paper, the performance of the proposed method is examined with the standard test functions [13]. The optimization problems used here are the minimization of the Rosenbrock function $(F 1)$, the Rastrigin function $(F 2)$, the Griewank function (F3), the Ridge function (F4). Table1 shows the test functions and its domains.

The Rosenbrock function is the unimodal function and it has dependence on its variables (nonseparable function). The Rastrigin function is the multimodal function and it has independence on its variables (separable function). The Griewank function is the multimodal function and it has dependence on its variables (nonseparable function). The Ridge function is unimodal function and it has
Table 1: Test functions and its domains

$$
\begin{aligned}
& F_{1}\left(x_{i}\right)=\sum_{i=1}^{n}\left[100\left(x_{1}^{2}-x_{i}\right)^{2}+\left(x_{i}-1\right)^{2}\right] \\
& -2.048 \leq x_{i}<2.048 \\
& F_{2}\left(x_{i}\right)=10 n+\sum_{i=1}^{n}\left(x_{i}^{2}-10 \cos \left(2 \pi x_{i}\right)\right) \\
& -5.12 \leq x_{i}<5.12
\end{aligned}
$$$$
\begin{aligned}
& F_{3}\left(x_{i}\right)=1+\sum_{i=1}^{n} \frac{x_{i}^{2}}{4000}-\prod_{i=1}^{n}\left(\cos \left(\frac{x_{i}}{\sqrt{i}}\right)\right) \\
& -512 \leq x_{i}<512
\end{aligned}
$$

$$
\begin{aligned}
& F_{4}\left(x_{i}\right)=\sum_{i=1}^{n}\left(\sum_{j=1}^{i} x_{j}\right)^{2} \\
& -64 \leq x_{i}<64
\end{aligned}
$$

dependence on its variables (nonseparable function).

The unimodal function has only one global optimum. On the other hand, the multimodal function has many local optima, but only one global optimum.

\subsection{Experimental setting}

In this paper, we compare the performance of the proposed method with the UNDX. An experimental setting is shown in Table2. On the unimodal functions $(F 1, F 4)$, number of individual is 300 . On the multimodal functions $(F 2, F 3)$, number of individual is 600 . On all functions, number of variable is 20 , number of applying crossover (UNDX) is 200 , number of generation is 30000 , parameters of UNDX $\sigma_{\xi}$ is $1 / 2$ and $\sigma_{\eta}$ is $0.35 / \sqrt{n}$. The initial population is generated randomly following the uniform distribution inside the region described in Table1. All experiments were carried out by changing the seed for random number.

\subsection{Simulation results}

Table 3 shows the average evaluations where the global optimum is discovered and the number of convergence times. These results are the average of 20 trials. Fig $4,5,6,7$ show the histories of the 
Table 2: Experimental Setting

\begin{tabular}{l|c}
\hline \hline Number of individuals & $300(F 1, F 4) / 600(F 2, F 3)$ \\
\hline Number of variables & 20 \\
\hline Number of max generations & 30000 \\
\hline Crossover & UNDX / CDX \\
$\sigma_{\xi}$ & $1 / 2 /-$ \\
$\sigma_{\eta}$ & $0.35 / \sqrt{n} / 1 / \sqrt{n}$ \\
Number of crossover time & 200 \\
\hline
\end{tabular}

Table 3: The average evaluations where the global optimum is discovered and the number of convergence times

\begin{tabular}{|c|c|c|c|}
\hline & Crossover operator & Average evaluations & Convergence times \\
\hline \hline Rosenbrock & UNDX & 6000000 & $0 / 20$ \\
function $(F 1)$ & Proposed method & 3289200 & $20 / 20$ \\
\hline Rastrigin & UNDX & 5215020 & $20 / 20$ \\
function $(F 2)$ & Proposed method & 3913960 & $20 / 20$ \\
\hline Griewank & UNDX & 4936230 & $20 / 20$ \\
function $(F 3)$ & Proposed method & 3106440 & $20 / 20$ \\
\hline Ridge & UNDX & 4951070 & $20 / 20$ \\
function $(F 4)$ & Proposed method & 1512030 & $20 / 20$ \\
\hline
\end{tabular}

best function value of the proposed method. Fig8, $9,10,11$ show the histories of the best function value of the UNDX. In these figures, the $y$ axis shows the function value, and the $x$ axis shows the generation. The $y$ axis is also $\log$-scale.

On the results (Table3, Fig4, Fig8) of the test function $F^{\prime} 1$, this function is unimodal function but it is nonseparable function, it is difficult for GA to find the optimal solution. However, the proposed method can find the optimal solution in all trials. In addition, the proposed method has the average evaluations where the global optimum is discovered less than the UNDX.

Secondly, on the results (Table3, Fig5, Fig9) of the test function $F 2$, this function is separable function but it is multimodal function and it has many local optima around global optimum. The proposed method also has the average evaluations where the global optimum is discovered less than the UNDX. In addition, the proposed method can stably search solution.

Then, on the results (Table3, Fig6, Fig10) of the test function F3, this function is multimodal function and it is noseparable function, it is difficult for GA to find optimal solution. The pro- posed method has the average evaluations where the global optimum is discovered less than the UNDX.

Finally, on the results (Table3, Fig7, Fig11) of the test function $F 4$, this function is unimodal function but it is nonseparable function. The proposed method has the average evaluations where the global optimum is discovered less than the UNDX.

We summarize all experimental results. The proposed method can find optimal solution in all trials. And, the proposed method has the average evaluations where the global optimum is discovered less than the UNDX. And, the proposed method can stably search solution. Especially, in the nonseparable functions $\left(F^{\prime} 1, F^{\prime} 3, F^{\prime} 4\right)$, the proposed method shows the good performance.

\section{Conclusion}

In this paper, we proposed the center distribution crossover (CDX) as a new crossover operator for real-coded GA. The performance of the CDX was evaluated with standard test functions. The performance of the proposed method was compared with the UNDX. Simulation results suggested that 
the CDX has high accuracy and processing speed. Especially, in the nonseparable functions ( $F 1, F 3$, $F 4$ ), the proposed method shows the good performance.

In the near future, we try to compare the proposed method with another crossover operators, apply to another optimization problems.

\section{References}

[1] D.E.Goldberg, "Genetic Algorithm in Search, Optimization and Machine Learning," Addison-Wesley, 1989

[2] L.Davis, "The Handbook of Genetic Algorithm," Van Nostrand, 1990

[3] J.H.Holland, "Adaptation in natural and artificial systems," The University of Michigan Press, Ann Anbar, 1975

[4] M.Yamamura, S.Kobayashi, "Toward Application Methodology of Genetic Algorithms," Journal of the Japanese Society for Artificial Intelligence, Vol.9, pp. 506-511, 1994, in Japanese

[5] H.Kitano, "Genetic Algorithm," Sangyoutosyo, 1993, in Japanese

[6] I.Ono, M.Yamamura, S.Kobayashi, "RealCoded Genetic Algorithms and Their Applications," Journal of the Japanese Society for Artificial Intelligence, Vol.15, pp. 259-266, 2000, in Japanese

[7] L.J.Eshelman and J.D.Schaffer, "Real Coded Genetic Algorithms and Interval Schemata, Foundations of Genetic Algorithms 2, pp. 187-202, 1993

[8] I.Ono, H.Satoh, S.Kobayashi, "A Real-Coded Genetic Algorithm for Function Optimization Using the Unimodal Normal Distribution Crossover," Journal of the Japanese Society for Artificial Intelligence, Vol.14, pp. 11461155, 1999, in Japanese

[9] H.Kita, I.Ono and S.Kobayashi, "Theoretical Analysis of the Unimodal Normal Distribution Crossover for Real-coded Genetic algo- rithm," Transactions of the Society of Instrument and Control Engineers, 34-8, pp. 13331339, 1999, in Japanese

[10] H.Kita, I.Ono, S.Kobayashi, "Multi-Parental Extension of the Unimodal Normal Distribution Crossover for Real-Coded Genetic Algorithm," Transactions of Society of Instrument and Control Engineers, Vol.36, pp. 875-883, 2000 , in Japanese

[11] H.Satoh, M.Yamamura, S.Kobayashi, "Minimal Generation Gap Model for GAs considering Both Exploration and Exploitation," Proceedings of IIZUKA'96, pp. 494-497, 1996.

[12] H.Satoh, I.Ono and S.Kobayashi, "A New Generation Alternation Model for Genetic Algorithms and Its Assessment," Journal of Japanese Society for Artificial Intelligence, 12-5, pp. 734-744, 1997, in Japanese

[13] D.Whitley, K.Mathias, S.Rana, and J.Dzubera, "Building better test functions," Proceedings of the 6th International Conference on Genetic Algorithms, pp. 239-246, 1995 


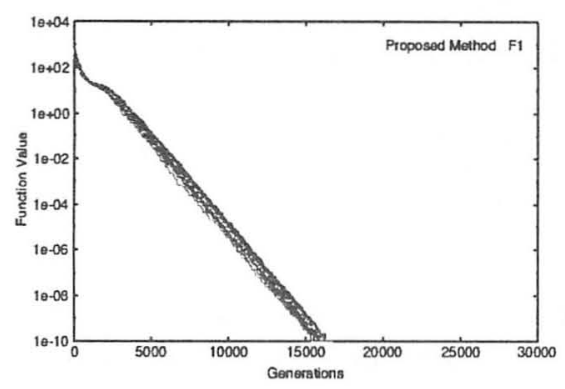

Fig 4: History of best function value in $F 1$

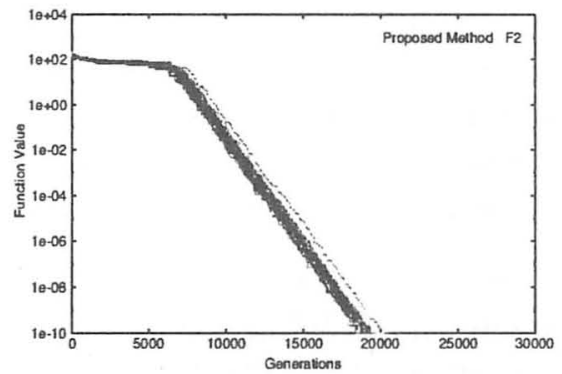

Fig 5: History of best function value in $F 2$

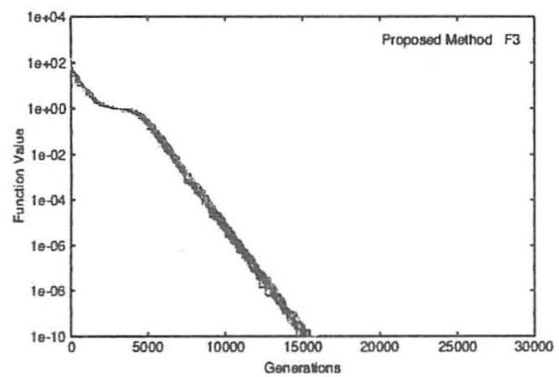

Fig 6: History of best function value in $F 3$

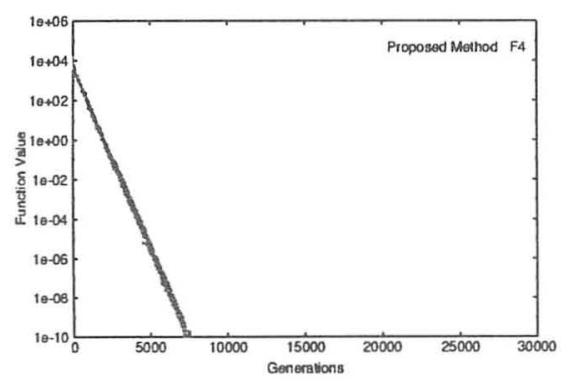

Fig 7: History of best function value in $F 4$

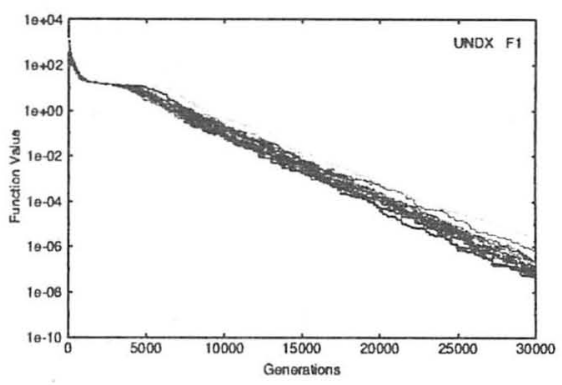

Fig 8: History of best function value in $F 1$

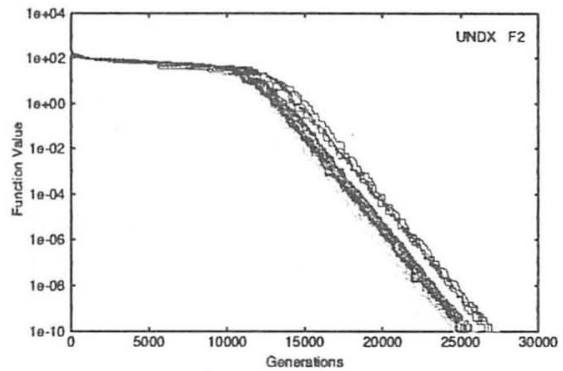

Fig 9: History of best function value in $F 2$

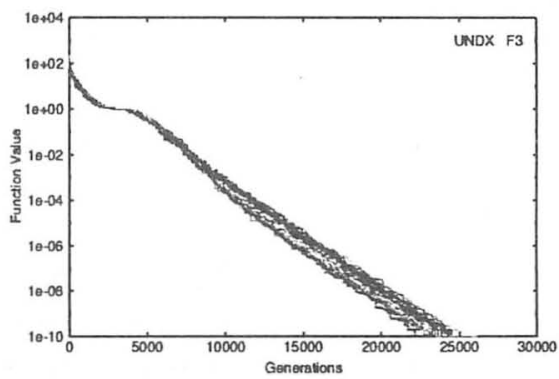

Fig 10: History of best function value in $F 3$

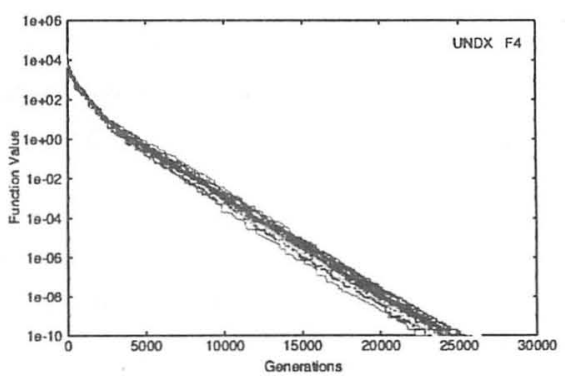

Fig 11: History of best function value in $F 4$ 